\title{
Resonance Capture of Neutrons in Infinite Homogeneous Media†
}

\author{
By R. SCHERMER \\$ AND N. CORNGOLD\| \\ I Massachusetts Institute of Technology, Cambridge, Mass. \\ I| Brookhaven National Laboratory, Upton, New York
}

MS. received 10th November 1958

Abstract. In a previous paper, a variational principle was introduced for $1-p$, the capture probability for neutrons slowing down in a homogeneous medium of infinite extent. In the present paper, the variational principle is used together with simple but accurate trial functions to obtain expressions for (i) corrections to the commonly used 'narrow resonance' formula for capture and (ii) interference effects in the capture of neutrons by closely spaced resonances.

\section{$\$ 1$. INTRODUCTION}

$T^{N}$ studying the slowing down of neutrons in, say, homogeneous media of infinite extent one is quite interested in the probability that a neutron will be captured by a nucleus of the moderating material during the slowing down process. A typical slowing-down medium might consist of a mixture of light moderator (hydrogen, carbon, ...) and heavy fertile or fissile material (thorium, uranium,...). The capture probability will be related to the neutron absorption cross section of the heavy nucleus. This cross section shows in general a characteristic resonance structure, and the existing analytical approximations for the capture probability take the effect of this violent variation into account only poorly (see for example Sampson and Chernick 1957).

The most popular analytical expression is based on the narrow resonance dpproximation, in which it is assumed that a given resonance covers such a narrow energy range that a single scattering collision with any nucleus in the medium will degrade the neutron energy below the resonance. This approximation leads to a neutron collision density through the resonance that is identical with the unperturbed, asymptotic density. It does not take into account the finite width of the resonance or the possibility of interference between resonances. A second approximation, called 'infinite absorber' denies the heavy nucleus the power to moderate neutrons (heavy nuclear mass tending to $\infty$ ), so that only collisions with the light nuclei can knock nucleons out of the resonance. This last approximation is useful for the broad resonances which occur at the lowest energies. It is not difficult to see that most resonances encountered satisfy neither the narrow resonance nor the infinite absorber conditions.

In this paper we use a quite accurate variational technique, introduced by one of us (Corngold 1957) in an earlier paper. We obtain analytical results for corrections to the narrow resonance formula in single resonances and for the interference effects in capture by closely spaced resonances.

† Work performed under the auspices of the U.S. Atomic Energy Commiation.

Research Assistant, Brookhaven National Laboratory, Summer, 1958.

PROC. PHYS. 3OC. LXXIII, 4 


\section{§ 2. Mathematical Development}

The variational expression for the resonance capture $1-p$, is expressed in terms of the functional $J\left[\phi, \phi^{+}\right]$, where

$$
\begin{aligned}
& J\left[\phi, \phi^{+}\right]= \\
& \int_{0}^{\infty} d u \sum_{s} g_{s}(u) \phi^{+}(u) \phi(u)+\int_{0}^{\infty} d u \sum_{l} g_{l}(u) \phi^{+}(u) \int_{0}^{u} d u^{\prime} \sum_{s} \psi_{s}\left(u-u^{\prime}\right) g_{s}\left(u^{\prime}\right) \\
& \int_{0}^{\infty} d u \sum_{l} g_{l}(u) \phi(u) \int_{0}^{\infty} d u^{\prime} \sum_{s} g_{s}\left(u^{\prime}\right) \phi^{+}\left(u^{\prime}\right)
\end{aligned}
$$

and the symbols have the meaning: the lethargy $u=\ln \left(E_{0} / E\right)$ where $E_{0}$ is the source energy and $E$ the energy variable; $g_{s}(u)=h_{9}(0)-h_{4}(u)$, where $h_{s}(u)$ is the probability that a collision at lethargy $u$ is a scattering collision with an $s$-type nucleus,

$$
h_{s}(u)=\sum_{s}^{\mathrm{scat}}(u) / \Sigma(u)
$$

and $\Sigma^{\text {scat }}, \Sigma$ are respectively macroscopic scattering and total cross sections. $\bar{\psi}_{s}$ is the collision density solution to an idealized slowing-down problem in the mixture under consideration; it obtains when all the $g_{s}(u) \equiv 0$ and neutrons produced by a source at $u=0$ make their first collision with a nucleus of type $s$. $\xi_{\text {av }}$ has its customary meaning. The functional $J\left[\phi, \phi^{+}\right]$is equal to $1 /\left[\xi_{\text {av }}(1-p)\right]$ when $\phi(u)$ is the collision density solution to the full slowing-down problem and $\phi^{+}(u)$ is stationary with regard to small changes $\delta \phi, \delta \phi^{+}$in these solutions.

From the form of the equations defining $\phi, \phi^{+}$(Corngold 1957), it appears that $\phi_{0}=\phi_{0}{ }^{+}=1 / \xi_{\mathrm{av}}$ is a good first guess at trial functions. Insertion of this value into (1) leads to what we shall call $(1-p)_{\text {var. }}$ That this value is a considerable improvement over the narrow resonance value-which we obtain by discarding the double integral in (1) and again choosing $\phi_{0}=\phi_{0}{ }^{+}=1 / \xi_{\mathrm{av}}$-is apparent from the structure of the double integral and is borne out by actual numerical computation. The last statement holds also for the broad, low energy resonances where the variational result lies close to the infinite absorber results. For convenience we rewrite (1), with the choice discussed above for $\phi$ and $\phi^{+}$, as

$$
\begin{gathered}
\frac{(1-p)_{\mathrm{var}}}{(1-p)_{\mathrm{NR}}}=\rho_{0}=\frac{1}{1+\chi} \quad x=I / \int_{0}^{\infty} \sum g_{s}(u) d u \\
I=\int_{0}^{\infty} \sum_{l} g_{l}(u) d u \int_{0}^{u} d u^{\prime} \sum \psi_{s}\left(u-u^{\prime}\right) g_{s}\left(u^{\prime}\right), \\
(1-p)_{\mathrm{NR}}=\frac{1}{\xi_{\mathrm{av}}} \int_{0}^{\infty} \sum g_{s}(u) d u .
\end{gathered}
$$

The error in the narrow resonance formula $(2 c)$ is due to the replacement of $\phi(u)$ by its asymptotic form $1 / \xi_{\mathrm{av}}$ in regions where it may deviate considerably from this value. By its stationary nature, the variational principle is much less sensitive to such variations in $\phi(u)$.

An analytical treatment of equations (2) is made difficult by the discontinuous nature of the functions $\bar{\psi}_{*}$ and the fact that the investigation involves integration over domains containing several singularities of $\bar{\psi}_{*}$. Thus direct substitution of $\psi_{a}$ into the double integral $(2 b)$ leads to exceedingly complicated algebra. We have found the double integral to be much more tractable if handled by 
Laplace transform methods. Indeed, for the resonance shapes we consider the variational expression may be put into a form such that effects occurring in successive collision intervals may be clearly identified.

Absorption in a single, isolated resonance in a system of hydrogen moderator and some heavier resonance absorber will now be discussed in detail. The result will then be generalized to heavier moderators and to the question of interference between two resonances lying close together. In all cases we will assume a simple, yet physically representative, shape for $g_{s}(u)$. The results will be expressible in terms of a double series. The important parameters of this series are $\lambda$, a quantity to be defined later and related to $\xi_{\mathbf{a v}}$; the width of the resonance; the spacing in the interference problem; and $\Sigma_{s} g_{s} /\left(1-\alpha_{s}\right)$, which characterizes the strength of the resonance.

Numerical results will be presented for the correction to the narrow resonance result, as predicted by the variational principle, for resonances of the chosen shape. These may be shown to be upper bounds to the corrections for real resonances.

\section{\$3. Single Resonance Absorption}

We now seek to evaluate $(2 b)$ for the case of absorption by one isolated resonance. To this end we write the

$$
\int_{0}^{u} \sum_{s} g_{s}\left(u^{\prime}\right) \psi_{s}\left(u-u^{\prime}\right) d u^{\prime}
$$

appearing in $(2 b)$ as the Laplace inverse of its Laplace transform

$$
\int_{0}^{u} \sum_{s} g_{s}\left(u^{\prime}\right) \Psi_{s}\left(u-u^{\prime}\right) d u^{\prime}=\frac{1}{2 \pi i} \int_{\Gamma-\imath \infty}^{\Gamma+i \infty} \mathrm{e}^{u \eta} \mathrm{L}\left\{\int_{0}^{u} \sum_{s} g_{s}\left(u^{\prime}\right) \Psi_{s}\left(u-u^{\prime}\right) d u^{\prime}\right\} d \eta .
$$

where the Laplace transform is defined by

$$
\mathrm{L},\{b(u)\}=\int_{0}^{\infty} \mathrm{e}^{-u \eta} b(u) d u=\tilde{b}(\eta) .
$$

The contour is any line lying to the right of all singularities of the integrand in the $\eta$ plane. We insert $(3)$ in $(2 b)$ and interchange the order of integration whence

where

$$
I=\frac{1}{2 \pi i} \int_{\Gamma-i \infty}^{\Gamma+i \infty} \sum_{i} \tilde{g}_{1}(-\eta) \sum_{i} \tilde{g}_{s}(\eta) \tilde{\psi}_{s}(\eta) d \eta
$$

$$
\tilde{g}_{s}(-\eta)=\int_{0}^{\infty} g_{s}(u) \mathrm{e}^{u \eta} d u
$$

and $\Psi_{*}(\eta)$ may be written in terms of the scattering kernel $K_{*}(\eta)$ which characterizes the scattering by an s-type nucleus.

$$
\begin{gathered}
K_{s}(u)=\frac{\mathrm{e}^{-u}}{1-\alpha_{s}} q_{s} \geqslant u \geqslant 0, \quad q_{s}=\ln \left[\frac{\left(m_{s}+1\right)}{\left(m_{s}-1\right)}\right]^{2}=\cdots \ln \alpha_{s} \\
=0 \quad \text { elsewhere, } \\
\Psi_{H}(\eta)=\frac{\tilde{K}_{s}(\eta)}{1-\sum_{s} h_{s}(0) \tilde{K}_{s}(\eta)} .
\end{gathered}
$$

It is easily shown that $\lim _{u \rightarrow \infty} \psi_{*}(u)=1 / \xi_{\text {av }}$. 
The functions $g_{s}(u)$ describe the resonance and are different from zero only within the resonance. We shall describe our resonances by the simple shape:

$$
g_{s}(u)= \begin{cases}0 & u<0 \\ g_{s} & 0 \leqslant u \leqslant a \\ 0 & u>a\end{cases}
$$

We may take the resonance at $u=0$, without loss of generality, since the results are invariant under a translation of the origin of $u$.

The reader should note that in the realistic case of cross sections described by Doppler-broadened, Breit-Wigner resonance shapes $g_{s}(u)$ is indeed nearly constant over the energy range in which the cross sections have appreciable size. In a typical case a numerical calculation showed only a $10 \%$ drop in magnitude of $g_{8}$ in an energy interval in which the total cross section fell by a factor of twenty. Moreover, by stating the problem in terms of the $g_{s}$ we obtain results which are close to universal in that they are almost independent of the height of the cross section peak. As long as we choose and compare profiles for $g_{s}(u)$ whose widths are cut off at a certain number of practical widths, $\tilde{g}_{s}(\eta)$ will have no poles in the finite plane and the form of $g_{s}(u)$ modifies only the residues in our integral $(5 a)$. We have examined the consequences of using triangular and parabolic profiles and we obtain results that have functional form similar to those obtained with the rectangular profile, but with magnitudes that are somewhat smaller.

The terms 'resonance height' and/or 'resonance width' will henceforth refer to the characteristics of $g_{s}$. Using (4), $(5 b),(6)$ and $(7)$ in $(2 b)$ we find that $I=\frac{1}{2 \pi i} \int_{\Gamma-i \infty}^{\Gamma+i \infty} d \eta\left(\frac{\mathrm{e}^{a_{\eta}}-1}{\eta}\right)\left(\frac{1-\mathrm{e}^{-a \eta}}{\eta,}\right) \sum_{i, s} \frac{g_{l} g_{a}}{\Gamma-\alpha_{s}} \frac{1-\alpha_{s} \exp \left(-q_{s} \eta\right)}{1+\eta-\sum_{p} \frac{h_{p}(0)}{1-\alpha_{p}}\left\{1-\alpha_{D} \exp \left(-q_{p} \eta\right)\right\}}$.

The simplest non-trivial case is that of hydrogen $(s=1)$ mixed with some heavier resonance absorber $(s=2)$. In this case (8) may be expressed via the substitutions

as integrals of the form

$$
\lambda=\sum_{p} \frac{h_{p}(0)}{1-\alpha_{p}}-1, \quad y_{2}=\frac{h_{2}(0) \alpha_{2} q_{2}}{\left(1-\alpha_{2}\right)}, \nu=q_{2} \eta
$$

$$
I^{\prime}=\frac{1}{2 \pi i} \frac{q_{2}}{\lambda} \int_{\Gamma-i \infty}^{\Gamma+i \infty} \frac{\mathrm{e}^{p_{\nu}} d \nu}{\nu^{2}\left(\nu / y_{2}+\mathrm{e}^{-\nu}-1\right)},
$$

where $p$ takes the values $a / q_{2},-a / q_{2}, 0,\left(a-q_{3}\right) / q_{2},-\left(a+q_{2}\right) / q_{2},-q_{2} / q_{2}$. Note that the identity $-\lambda+y_{2} / q_{2}=0$ holds.

The integrals (9) are inconvenient to evaluate directly by the method of residues, since $\left(v / y_{2}+e^{-v}-1\right)^{-1}$ has a simple pole at the origin and an infinite set of complex-conjugate pole pairs in the left half-plane extending out to infinity. This portion of the denominator, it should be emphasized, is due to $\psi_{s}(\eta)$. The chosen functions $\tilde{g}_{s}(\eta), \tilde{g}_{s}(-\eta)$ have no singularities in the finite part of the plane.

Consider the expansion

$$
\left(\frac{\nu}{y_{2}}+\mathrm{e}^{-\nu}-1\right)^{-1}=\frac{y_{2}}{\nu} \sum_{k=1}^{\infty}\left(-y_{2}\right)^{k}\left(\frac{\mathrm{e}^{-\nu}-1}{\nu}\right)^{k} .
$$


This is readily shown to converge for $\mathscr{R} \nu>0$, since in this region $\left|\left(e^{-\nu}-1\right) / \nu\right|^{2}<1$ and $0<y_{2}<1$. This expansion has an interesting physical significance. It is essentially an expansion of $(6 b)$ in the form

$$
\tilde{\psi}_{s}(\eta)=\tilde{K}_{s}(\eta)\left[1+\sum_{s} h_{s}(0) \tilde{K}_{s}(\eta)-\left(\sum_{s} h_{s}(0) \tilde{K}_{s}(\eta)\right)^{2}+\ldots\right] \ldots \ldots
$$

which represents source neutrons making successively $1,2, \ldots$ collisions. On summing the successive integrands which result when $(10)$ is inserted into (9) we will get the contribution to the resonance capture due to neutrons making $1,2,3, \ldots$ collisions in the resonance.

Next we expand $\left(\mathrm{e}^{-\nu}-1\right)^{k}$ by the binomial theorem and interchange sums and integrals to obtain

$$
I^{\prime}=\frac{1}{2 \pi i} \frac{q_{2} y_{2}}{\lambda} \sum_{j=0}^{\infty} \sum_{k=j}^{\infty} \int_{\Gamma-i \infty}^{\Gamma+i \infty}(-1)^{\jmath}\left(y_{2}\right)^{k} \frac{\left(\begin{array}{l}
k \\
j
\end{array}\right) \mathrm{e}^{(p-j) \nu}}{\nu^{3}+k} d \nu .
$$

The sum over $j$ is also physically interesting. Each term represents, as will become more apparent when the integration is performed, the full contribution to the capture due to a region of width $q_{2}$ at distance $j q_{2}$ from the source.

The integral (12) will now be evaluated in the customary manner by closing the straight line path with a curved contour whose radius is made to approach infinity, and upon which the integral approaches zero. When $p-j<0$ the closed contour extends to the right of the original path, when $p-j>0$ it extends to the left. In the case $p-j \leqslant 0$ the contour encloses no singularities of the integrand and $I^{\prime}=0$. For $p-j>0$ we have the residue of a pole of order $3+k$ at the origin. This means that $j$ takes on a maximum value equal to the largest integer less than $p$, symbolized as $[N]<p$. Performing the integration for $y_{2}$ and expanding $\left(\begin{array}{c}k \\ j\end{array}\right)$ we get as a result:

$$
I^{\prime}=\frac{1}{\lambda^{2}} \sum_{j=0}^{[N]<p} \sum_{k=j}^{\infty} \frac{(-1)^{j}\left[\lambda q_{2}(p-j)\right]^{k+2}}{j !(k-j) !(k+2)(k+1)}
$$

From the values that $p$ takes on and the above discussion regarding choice of contours, we see that only two values of $p$ gives non-zero contributions. $p=a \mid q_{2}, a>0 ; p=a / q_{2}-1, a>q_{2}$. (8) then may be rewritten as

$$
I=\left(\sum_{l} g_{l}\right)\left[I^{\prime}(a) \sum_{i} \frac{g_{s}}{1-\alpha_{s}}-\frac{y_{2} \alpha_{2}}{1-\alpha_{s}} I^{\prime}\left(a-q_{2}\right)\right]
$$

where it is understood that the second term vanishes if $a<q_{2}$. It may readily be verified that (13) may also be written as the single sum:

$$
\lambda^{2} I^{\prime}=\sum_{j=0}^{i N}\left\{\frac{\left(-T^{j}\right)}{j !} \frac{d^{j}}{d T^{j}}\left[\frac{\mathrm{e}^{T}-1-T}{T^{j}}\right]\right\}_{T-v_{i}[p-j]}
$$

The sums converge very rapidly with $j$. Four terms $(j \leqslant 3)$ give accuracy to approximately $0.1 \%$ for the largest argument $y_{\mathrm{g}} p$ we have calculated $\left(y_{2} p=0.94\right.$, corresponding to a resonance $20 q_{8}$ wide in a $10: 1, \mathrm{H}-983 \mathrm{U}$ mixture). This is equivalent to the usual statement in the literature that $\psi_{s}(u)$ reaches its asymptotic value within $3 q_{\mathrm{g}}$ of sources or sinks.

With these results, we may write $(2 a)$ in a particularly instructive form:

$$
\rho_{0}=\left[1+a \sum_{i} \frac{g_{B}}{1-\alpha_{a}}\left(\frac{I^{\prime}(a)}{a^{2}}\right)-\alpha_{2} a \frac{g_{2}}{1-\alpha_{8}}\left(\frac{I^{\prime}\left(a-q_{8}\right)}{a^{2}}\right)\right]^{-1} \text {. }
$$


Here

$$
\begin{aligned}
\frac{I^{\prime}(a)}{a^{2}} & =F(\lambda a) & \text { for } & a \geqslant 0, \\
& =0 & \text { for } & a<0 .
\end{aligned}
$$

For example,

$$
F(\lambda a)=\frac{1}{2 !}+\frac{\lambda a}{3 !}+\frac{(\lambda a)^{2}}{4 !}+\ldots \text { when } a \leqslant q_{2} .
$$

The important quantities in (16) are: $g_{s} a /\left(1-\alpha_{s}\right), s=1,2$, whose magnitudes are less than 1 in all practical cases, and are parameters describing the effective strength of the perturbing resonance, and the function $F(\lambda a)$, which depends upon the nature of the mixture and whose value is a measure of the average number of collisions required to carry a neutron through the resonance. As we shall see later, a similar equation results in the case of moderation by uranium and arbitrary moderator, so long as $a<q_{1}$. The strength parameters are isolated neatly in these calculations and since they are often small an expansion of (16) in terms of them yields a rapidly converging sequence of corrections to the narrow resonance result.

\subsection{Non-Hydrogenous Systems}

The case of systems in which the heavy absorber is mixed with moderator other than hydrogen is more complicated algebraically than the previous case but follows the same lines. The last term in the denominator of $\vec{\psi}_{a}(\eta)$ becomes

$$
\sum_{s} \frac{h_{8}(0) \alpha_{s}}{1-\alpha_{s}} \mathrm{e}^{-\alpha_{s} \eta} \quad \text { instead of } \frac{h_{2}(0) \alpha_{2}}{1-\alpha_{2}} \mathrm{e}^{-q_{2} \eta}
$$

The denominator is first expanded as in (10). Two binomial expansions are now necessary; one in $j$, measuring distances in units of $q_{2}$, and one in $i$, measuring distances in units of $q_{1}$. The only non-zero terms contributing to the integral (8) are for $p=a / q_{2}, a>0 ; p=\left(a-q_{2}\right) / q_{2}, a>q_{2} ;$ and $p=\left(a-q_{1}\right) / q_{2}$, $a>q_{1}$. For practical moderators (carbon or lighter), the last case would represent an unrealistically wide resonance and may be ignored. With this proviso (13) becomes

$$
I^{\prime}(p)=\frac{1}{\lambda^{2}} \sum_{j=0}^{\infty} \sum_{k=j}^{\infty}(-1)^{j} \frac{\left[\lambda q_{2}(p-j)\right]^{k+2}}{j !(k-j) !(k+1)(k+2)}\left(\frac{y_{2}}{\lambda q_{2}}\right)^{j}, \quad p<\frac{q_{1}}{q_{2}} .
$$

$y_{2}$ and $\lambda$ are as defined previously, but they now obey the relation

$$
\lambda-\frac{y_{2}}{q_{2}}-\frac{y_{1}}{q_{1}}=0, \text { where } y_{1}=\frac{h_{1}(0) \alpha_{1} q_{1}}{1-\alpha_{1}} .
$$

For mixtures containing hydrogen $y_{1}=0$, and these expressions reduce to ones quoted earlier. $I^{\prime}$ is still related to $I$ by $(14)$ and the discussion following (16) applies here as well.

In order to consider the expression for $\rho_{0}$ in some detail, we must examine the quantities $h_{g}$ and $g_{g}$. In all cases $0 \leqslant h_{g}(u) \leqslant 1, \Sigma_{g} h_{s}(0)=1$ and $-1 \leqslant g_{g}(u) \leqslant 1$. If we assume that the light material does not capture neutrons we can deduce further that $g_{1}(u)>0$, while $g_{9}$ can have either sign. Since $1 /\left(1-\alpha_{2}\right)>1 /\left(1-\alpha_{1}\right)$ the sign of $\Sigma_{a} g_{a} /\left(1-\alpha_{s}\right)$ and of the correction depends largely upon the sign of $g_{g}$. From its definition it will be seen that if $g_{2}>0,0<g_{2}<h_{2}(0)$, and is generally quite small. However, when $g_{g}$ is negative its magnitude may be of the order of unity. Therefore the corrections for resonances characterized by $g_{8}>0$, which will henceforth be called absorption resonances, will be less than those 
of the second type (scattering resonances). One should note that the definition of scattering resonance given here encompasses resonances characterized approximately by $\Gamma_{n} / \Gamma>h_{2}(0)$, which may include resonances with $\Gamma_{\gamma}>\Gamma_{\mu}$.

Figure 1 shows the corrections $\rho_{0}$ to the narrow resonance as a function of resonance width for three systems, two hydrogen-moderated and one carbonmoderated.

We have taken nominal potential scattering cross sections as $\sigma_{\mathrm{C}}(0)=5 b$; $\sigma_{\mathrm{H}}(0)=20 b ; \sigma_{\mathrm{U}}(0)=10 b$. The scattering resonance chosen has peak resonance cross section $\sigma_{2}^{\text {scat }}$ (res $)=600 b, \sigma_{2}^{a}$ (res) $=1000 b$, while the absorption resonance has $\sigma_{2}^{\text {scat }}($ res $)=0, \sigma_{2}^{a}($ res $)=1000 b$. Moderators are compared at equal values

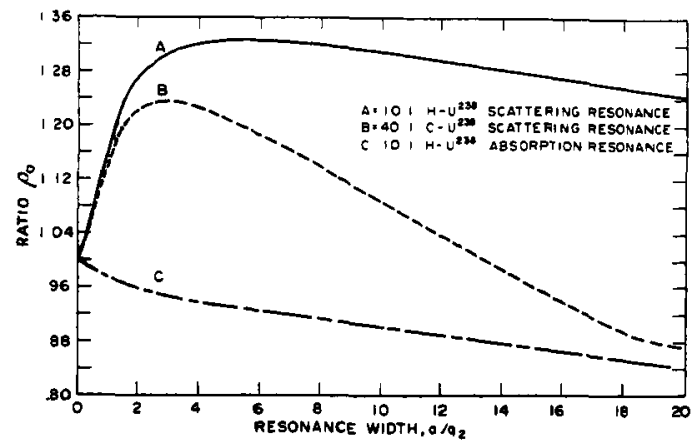

Figure 1. Ratio of variational to narrow resonance calculations of capture in single resonance in variety of systems. Resonance width is measured in lethargy units of size $q_{2}$.

of $h_{s}(0)$ (potential scattering per heavy atom). Thus $40: 1, \mathrm{C}-\mathrm{U}$ is to be compared with $10: 1, \mathrm{H}-\mathrm{U}$.

Note that for scattering resonances $\rho_{0}>1$ since the narrow resonance result ignores the build-up of the collision density in the resonance, which would lead to greater capture. For absorption resonances $\rho_{0}<1$ since the narrow resonance result ignores the depletion due to absorption, which would lead to lower capture.

\section{\$4. INTERFERENCE BETWEEN Resonances}

We now consider two resonances, of width $a$ and $b$, each with $g_{s}(u)$ of the rectangular shape discussed above, with edges located at $u=0, a, a+l, a+b+l$.

If the transform of such a function is inserted into $(5 a)$ we may separate the integrand into four groups of terms with the following physical significance. Two are equivalent to (8) and represent the effect of the two resonances acting independently. Two are interaction terms, one of which represents the effect of the second resonance on the first, which we expect should be zero. Indeed it is found that the normal form (9) of this integral contains only integrands with $p \leqslant 0$, all of which integrate to zero, following an earlier discussion. The fourth group we call the interference integral $J$. It has the form

$$
\begin{aligned}
J= & \frac{1}{2 \pi i} \sum_{r} g_{r b} \sum_{a} \frac{g_{s a}}{1-\alpha_{a}} \\
& \times \int_{\Gamma-i \infty}^{\Gamma+i \infty} d \eta \frac{\left(1-\alpha_{a} \mathrm{e}^{-l i m}\right)\left(\mathrm{e}^{(a+b+l)}+\mathrm{e}^{l \eta}-\mathrm{e}^{(a+l) m}-\mathrm{e}^{(b+l m)}\right)}{\eta^{2}\left[1+\eta-\sum_{1} \frac{h_{a}(0)}{1-\alpha_{a}}+\sum_{0} \frac{h_{d}(0) \alpha_{a}}{1-\alpha_{a}} \mathrm{e}^{-\alpha_{\Delta} \eta}\right]} .
\end{aligned}
$$


Note that if $a$ or $b$ equals zero, the integral vanishes, as it should. If $l=0$, the numerator becomes $\mathrm{e}^{(a+b) m}-\mathrm{e}^{a \eta}-\mathrm{e}^{b \eta}+1$. The last term does not contribute. the second and third terms cancel the contributions from the integrals which represent $a$ and $b$ acting alone, and we are left with the contribution of a resonance of width $a+b$.

The integration is exactly that which we have performed previously. For the case of a general mixture

$$
\begin{array}{r}
J^{\prime}=\frac{1}{\lambda^{2}} \sum_{m=1}^{4}(-1)^{m} \sum_{l=0}^{\left[\mathrm{N}_{m}\right]<p_{m}} \sum_{h=j}^{\infty} \frac{(-1)^{j}\left[\left(p_{m}-j\right) \lambda q_{2}\right]^{k+2}}{j !(k-j) !(k+2)(k+1)}\left(\frac{y_{2}}{\lambda q_{2}}\right)^{j} p_{m}<\frac{q_{1}}{q_{2}}, \\
\ldots \ldots\left(p_{1}=\frac{a+l}{q_{2}}, \quad p_{2}=\frac{a+b+l}{q_{2}}, \quad p_{3}=\frac{b+l}{q_{2}}, \quad p_{4}=\frac{l}{q_{2}} \quad \ldots \ldots\right.
\end{array}
$$

where $J^{\prime}$ is related to $J$ in the same way as $I^{\prime}$ is related to $I(14)$. Note the $J^{\prime}$ is a sum of four series of the form (17). The sum over $m$ is an artifice to take care of the four terms in (18). (19a) holds if all $p_{m}<q_{1} / q_{2}$, certainly the case in hydrogen-moderated systems. New terms start to enter when any of the $p_{m}$ become greater than $q_{1} / q_{2}$. Note that the upper limit of the sum over $j$ may be different for the different $p_{m}$.

It may be shown that the series converges as $l$, and hence all the $p_{m}$ become very large. The result, which we term $J_{\infty}$, is equal to $\Sigma_{r} g_{r a} \Sigma_{s} g_{s b}\left(a b / \xi_{\mathrm{av}}\right)$ which is independent of $l$ as expected on physical grounds. $J_{\infty}$ is easily shown to be the portion of (18) due to the $\eta=0$ pole of $\psi_{s}(\eta)$, i.e. the asymptotic value $\psi(u)=1 / \xi_{\mathrm{av}}$ in the $u$-plane. It is related to the 'bite' taken out of the flux by the first resonance. We will subtract this portion from $J$ in the numerical work.

Finally, we exhibit the form of the correction in analogy with equations (2),

$$
\begin{gathered}
\quad \frac{(1-p)_{\mathrm{Var}}}{(1-p)_{\mathrm{NR}}}=\rho=\frac{1}{1+X+Y}, \\
X=\frac{I_{a}+I_{b}+J_{\infty}}{(1-p)_{\mathrm{NR}}}, \quad Y=\frac{J-J_{\infty}}{(1-p)_{\mathrm{NL}}} .
\end{gathered}
$$

$I_{a}$ and $I_{b}$ refer to (17) applied to resonances $a$ and $b$ respectively. For the interference studies we define $\rho_{1}=1 /(1+X)$ and use the parameter $\rho_{2}=\left(\rho-\rho_{1}\right) / \rho_{1}=-\rho_{1} Y /\left(1+\rho_{1} Y\right)$ to indicate the magnitude of the interference effect. $\rho_{2}$ is the ratio of captures due to interference to non-interference captures.

\section{\$5. Numerical Results}

The basic series (17) is very rapidly convergent. Convergence in $j$ has been previously discussed, and the series in $k$ converges like the exponential, as indicated by (15). Thus for any given system specified by $\lambda q_{2}$ and $y_{2}$ with resonance width or spacing specified by the $p_{m}$, it is a simple matter to generate the series by hand computation, or, as we have done, with a small computing machine.

Specific results are presented below in the form of figures. These are based, of course, on the rectangular profile chosen for $g_{A}(u)$. Examination of the consequences of refining the profile has convinced us that the results here presented are a slight overestimate of the valuen to be obtained for real systems. 


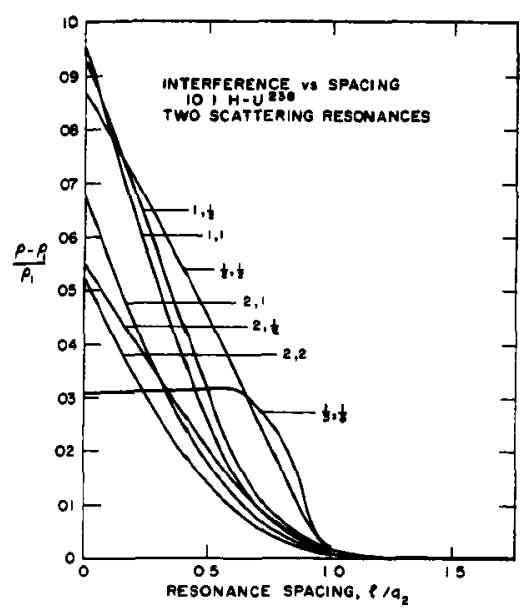

Figure 2. Ratio of captures ascribed to interference to captures obtained when the resonance spacing is made infinite. Resonance spacing is measured in lethargy units of size $q_{2}$.

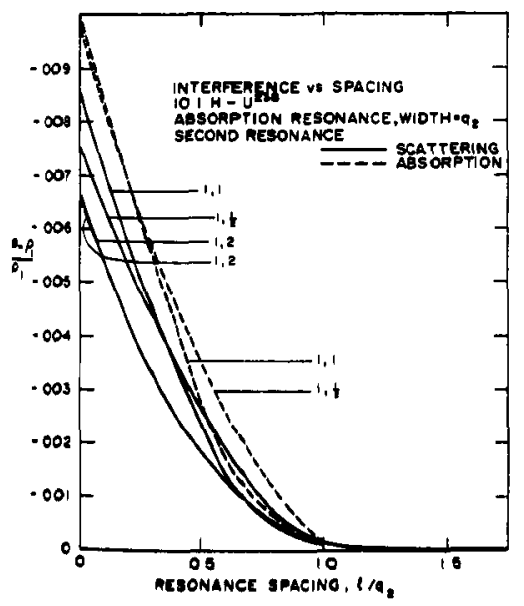

igure 4. As figure 2. Here an absorption resonance of fixed width acatters into a variety of second resonances.

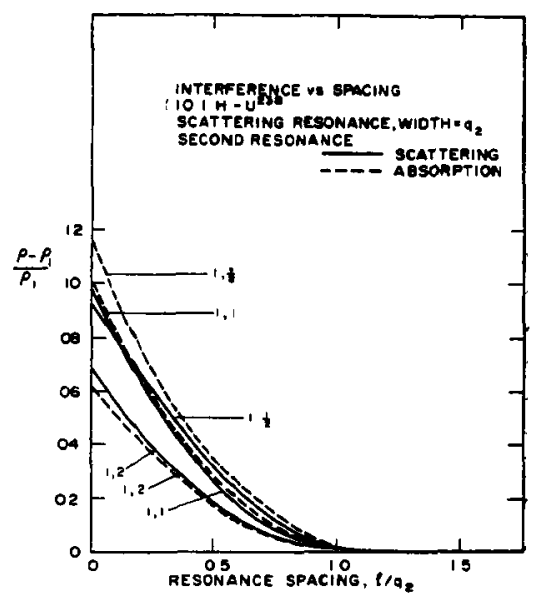

Figure 3. As figure 2. Here a scattering resonance of fixed width scatters into a variety of second resonances.

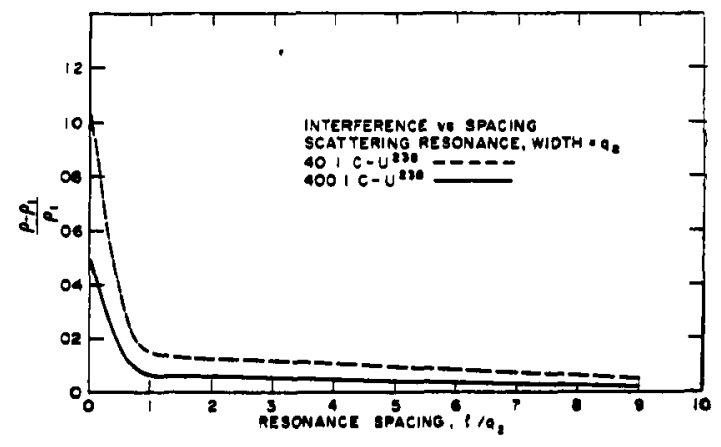

Figure 5. As figure 2. We consider two different mixtures of $\mathrm{C}-{ }^{30} \mathrm{U}$ and interference between two identical scattering resonances. 
Figure 1 deals with capture by a single resonance in typical $C-{ }^{238} \mathrm{U}, \mathrm{H}-{ }^{238} \mathrm{U}$ mixtures and displays the ratio $(1-p)_{\mathrm{var}} /(1-p)_{\mathrm{NR}}$ as a function of resonance width. Figures 2 to 4 display the interference effect $\rho_{2}$ as a function of resonance spacing $l / q_{2}$ for several systems. In figure 2 , two scattering resonances of varying width in a $10: 1 \mathrm{H}-{ }^{238} \mathrm{U}$ system are studied. The notation $(a, b)$ for each curve tells the width in units of $q_{2}$ of the first and second resonances respectively. In figures 3 and 4 , which also deal with a $10: 1 \mathbf{H}-{ }^{238} \mathrm{U}$ system, the first resonance is of fixed width $q_{2}$ and is of scattering, then absorption type, while the second resonance is of varying width and varying type. The sign of the interference effect is governed by the function $\Sigma_{q} g_{4} \psi_{4}$ and it is not difficult (Corngold 1957) to predict the result found in these figures, that perturbations in the collision

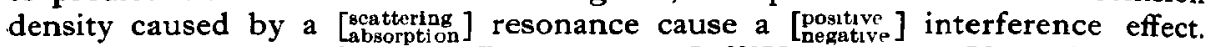
Figure 5 shows interference effects in two $C-{ }^{238} \mathrm{U}$ systems. Here the curves do not fall rapidly to zero because the effect of carbon scattering does not disappear for several $q_{1}$ from the resonance, and the calculations have only been done to points slightly less than $q_{1}$.

\section{\$6. Summary and Discussion of Results}

We believe that the following conclusions regarding real systems of moderator and resonance absorber may be drawn from our work.

1. The narrow resonance approximation may be very bad in any given resonance, especially one in which the resonance is of the order of or larger than $q_{2}$ and/or $\Sigma_{s} g_{d} /\left(1-\alpha_{s}\right)$ is significantly different from zero. Similar conclusions may be found in the studies of Spinney (1957) and Schermer (unpublished), the latter quoted partially by Corngold (1957). They deal with $1: 1 \quad \mathbf{H}-{ }^{238} \mathrm{U}$ mixtures, and thus it is not surprising that the corrections are even larger than those calculated above for a 10:1 mixture. The overall error in calculating $1-p$ using the narrow resonance approximation for all resonances may be comparatively small however due to cancellation of large errors of opposite sign. Such a calculation has been shown by Spinney (1957). The correction is positive for 'scattering' resonances, negative for 'absorption' resonances. The distinction of resonance type is based on the sign of $\Sigma_{*} g_{N} /\left(1-\alpha_{*}\right)$ generally, although nonhydrogenous moderators have other considerations. This is essentially Spinney's parameter in his 'modified narrow resonance' calculations.

2. The corrections increase with heavy atom concentration, although the dependence is not a strong one.

3. The (single resonance) correction decreases with moderator mass, the scattering cross section per heavy atom being held constant.

4. The corrections are not necessarily larger for resonances with larger peak cross sections. It is the fractional scattering probability which is important (this we have called $h_{*}$ ). However for the very small resonances encountered in the high energy range the corrections will be much smaller than those exhibited here. These are resonances in which the peak heights are of the order of the potential cross sections.

5. The interference portion of the correction term is quite small even at its largest values.

6. The interference at essentially zero spacing is a maximum for two resonances both on the order of $\frac{1}{2} q_{\mathrm{g}}$ to $q_{\mathrm{g}}$ wide. It is smaller for both narrower and wider resonances. 
7. The interference drops off with spacing more slowly for narrow resonances $\left(a<q_{2}\right)$ than for wide ones. For hydrogen moderator the interference is essentially zero for spacings $l>q_{2}$. The drop is less sharp with heavier moderators, but the interference is still small at spacings $l>q_{2}$.

8. The interference is greatest for a scattering resonance at the higher energy. It is completely negligible for an absorption resonance at the higher energy.

\section{ACKNOWLEDGMENT}

The authors acknowledge with thanks the computational assistance given them by Mr. William Bornstein.

\section{REFERENCES}

Corngold, N., 1957, Proc. Phys. Soc. A, 70, 793.

SAMpson, J., and Chernick, J., 1957, Progress in Nuclear Energy, Series I-Physics and Mathematics, 2, 223 (London: Pergamon Press).

SPINNEY, K. T., 1957, F. Nuclear Energy, 6, 53. 